Europe's Journal of Psychology, 6(4), pp. 93-109

www.ejop.org

\title{
Assessing movement imagery ability: Self-report questionnaires vs. performance-based tests
}

\author{
David Moreau \\ University of Lille 3, France \\ Dillard University, USA
}

Jérôme Clerc, Annie Mansy-Dannay, Alain Guerrien

University of Lille 3, France

\begin{abstract}
This study was designed to compare the relevance of self-report questionnaires and performance-based tests to assess movement imagery ability in sports. Participants included elite and novice athletes, from fencing, judo and wrestling, who completed a self-report, the Movement Imagery Questionnaire - Revised (MIQ-R; Hall \& Martin, 1997), and two performance-based tests, the Movement Imagery Specific Test (MIST), and the Mental Rotation Test (MRT; Vandenberg \& Kuse, 1978). There was no significant effect of the expertise variable on the MIQ-R performance, although the results yielded a positive effect of expertise on the MIST and on the MRT. Besides, results showed no correlations between the MIQ-R and the MIST, or between the MIQ-R and the MRT. However, we found a correlation between the MIST and the MRT. These findings are in line with research dissociating imagery measured by self-reports and spatial ability assessed through performance-based tests, and are discussed in terms of their implication in using self-report questionnaires in experimental psychology in general, and to assess movement imagery ability in sports in particular.
\end{abstract}

Keywords: movement imagery ability, visuospatial abilities, performance-based tests, self-report questionnaires, sport performance.

\section{Background}

Motor imagery can be defined as the result of conscious access to motor representations, normally non-conscious while performing an action (Jeannerod, 
1995). Thus, motor representations appear to be involved in different processes such as performing or visualizing an action, as well as watching one's performance, planning or verbalizing an action, and even observing an object commonly associated with a particular action (see Decety, 2002, for a review; Jeannerod, 1994, 2001). Research on the relationship between motor imagery and sport performance has been rather extensive and productive over the last few decades. The review of these studies has shown an effective enhancement of performance with the use of multiple imagery techniques (Driskell, Copper, \& Moran, 1994), consistent with research findings in a motor context (see Grèzes \& Decety, 2001, for a review). Also, different perspectives (first or third person) and modalities (mainly visual or kinesthetic), which should not be confused (Morris, Spittle, \& Watt, 2005), have been identified. Perspectives refer to whether imagery is experienced form inside or outside one's body, whereas modalities refer to the perceptual experience. Although first-person perspective and kinesthetic imagery, on one hand, and thirdperson perspective and visual imagery, on the other, are commonly associated with one another; recent work showed that third person perspective seems to favor kinesthetic modality (Callow \& Hardy, 2004).

Furthermore, it has been shown that motor imagery, regardless of perspective or modality, leads to better performance on various motor tasks, and that the combination of motor imagery and physical practice systematically shows an enhancement of motor performance, greater than or at least equal to physical practice alone (Feltz \& Landers, 1983; Driskell, Copper, \& Moran, 1994). Also, to optimize subsequent improvements, motor imagery characteristics should be close to those of physical execution (Roure et al., 1998).

Following these findings, cognitive psychology experiments and individual differences studies have found that imagery ability could influence motor performance (Yaguez et al., 1998), as well as motor accuracy (Lorey et al., 2010). In sports, research has shown that athletes could improve their imagery ability with an appropriate training focused on that particular purpose (Weinberg, 2008), suggesting that imagery ability, like spatial abilities (Lohman \& Nichols, 1990; Leone, Taine, \& Droulez, 1993), is partly genetically determined but also improvable with practice. Besides, recent studies have shown a relationship between motor practice and mental rotation processes (Jansen, Titze, \& Heil, 2009), and between elite performance in sports and mental rotation ability (Moreau, Mansy-Dannay, Clerc, \& Guerrien, submitted for publication), suggesting that motor practice and spatial abilities development are closely related. 
Thereby, experimenters in cognitive psychology, as well as in sport psychology, have worked on developing suitable tools and tests to measure imagery ability related to movements. To that end, Isaac, Marks and Russell (1986) designed the Vividness of Movement Imagery Questionnaire (VMIQ). Thereafter, Hall and Pongrac (1983) built the Movement Imagery Questionnaire (MIQ) to assess visual and kinesthetic movement imagery ability. This test is actually a self-report with 18 moves to execute and then visualize. Subjects are asked to rate their own performance on the visualization task based upon their own opinion, on a seven-point rating scale, varying from 'very easy to see/to feel' to 'very hard to see/to feel'. Validity and reliability of the MIQ have been confirmed by Hall, Pongrac and Buckolz (1985), and Atienza, Balaguer, and Garcia-Merita (1994). These authors found internal consistency coefficients of respectively .87 and .89 for the visual scale and .91 and .88 for the kinesthetic scale. Test-retest coefficients were identical in both studies (.83).

From the MIQ, Hall and Martin (1997) developed the Movement Imagery Questionnaire-Revised (MIQ-R), which is a shortened version including only eight items (four visual and four kinesthetic items) and suppressing difficult or redundant moves. The purpose was to reduce the testing time in order to favor larger studies. They found a significant correlation between both tests and concluded that the $M I Q-R$ is a relevant version of the MIQ (Hall \& Martin, 1997). Consequently, this test has been used by many experimenters and coaches in the sport field. Furthermore, studies showed that subjects with a good imagery ability assessed by self-reports learn new moves faster than subjects with low imagery ability (Goss, Hall, Buckolz, \& Fishburne, 1986), and that they are more accurate while executing precise moves (Hall, Buckolz, \& Fishburne, 1989), emphasizing the importance of assessing imagery ability among athletes, for instance.

However, this kind of test relies completely on participants' objectivity and selfperception of their own performance. In fact, the introspective nature of this test raises two experimental problems. Firstly, participants might not be willing to divulgate information about their imagery processes, purposely or because it would not meet the researcher's expectations. This can be conscious or non-conscious. This is obviously common to all self-report assessments, but it has to be taken into consideration when interpreting results. Secondly, and although participants might try to take the test as honestly as they can, there is still a part of mental imagery ability processes being possibly non-conscious (Morris, Spittle, \& Watt, 2005), and thus difficult if not impossible to access through self-perception. 
Moreover, performance-based tests have been popularly used in experimental psychology to measure spatial abilities. In fact, mental imagery and spatial abilities have been assessed through parallel, yet distinguished ways. Thus, research on mental imagery traditionally used questionnaire and vividness scales, whereas experimental designs assessing individual differences in spatial ability focused on performance-based tests (Hegarty \& Waller, 2005). Factorial studies constantly show no correlation between the former and the latter (Richardson, 1977, 1983; Kosslyn, Brunn, Cave, \& Wallach, 1984; Poltrock \& Agnoli, 1986). However, recent study by Burton and Fogarty (2003), has found that the relationship between self-report measurements and spatial tests is stimuli-dependent. Thus, they reported a relationship between these two types of measures when the items to imagine are similar to those involved in spatial ability tasks. These results are consistent with Dean and Morris findings (1991) showing that imagery questionnaires based on geometric figures are more likely than traditional self-reports to yield correlations with spatial tasks that contains the same kind of shapes. In fact, traditional self-report questionnaires seem to tap into different processes, favoring the recall of familiar objects or scenes previously stored into long-term memory.

\section{Research question}

The aim of the present study is to determine the relevance of self-report questionnaires and performance-based tests for assessing movement imagery ability in sports. On a broader perspective, we will also discussed self-reports questionnaires' use in experimental psychology. To this purpose, we worked with athletes from three different sports, well-known for their important training focused on mental repetition: fencing, judo and wrestling. Athletes were either elite lat least ranked at the Olympics, World championship, and/or European championship) or novices (no significant result in competition). Indeed, elite athletes show outstanding motor processes and though working with them seemed quite relevant when assessing motor representations modalities. We compared results from three different tests: a self-report questionnaire, the MIQ-R, and two performance-based tests, the Movement Imagery Specific Test (MIST, Moreau, Mansy-Dannay, Clerc, \& Guerrien, under review) and the Mental Rotation Test (MRT, Vandenberg \& Kuse, 1978).

\section{Method}

Participants

Sixty participants (mean age $=22.8$ years; range: 18-29) volunteered to take part in this study. Half of them were elite athletes, who had participated in an international 
championship. They were recruited in several federal sport institutions to which we were granted access. The remaining half was composed of novice athletes, who had practiced their sport for one year or less, and mostly had not participated in any competition. They were recruited in local affiliated clubs. There was no compensation of any kind for participating in the study. The distribution for each of the three sports was as follows: twenty athletes (five elite males, five elite females, five novice males, five novice females). Particular precautions were taken to have comparable mean ages between groups (elites mean age $=22.3$ years, novices mean age $=23.3$ years), since this factor can significantly affect imagery ability. In fact, spatial ability performance has been shown to increase until the age of 18 and drop down radically after 40 (Schroeder \& Salthouse, 2004; Kirasic \& Allen, 1985). Besides age, we ensured that athletes were not involved in particular activities related to higher spatial abilities, such as particular jobs (see Halpern \& Collaer, 2005; and Hegarty \& Waller, 2005, for reviews), or videogames playing (Boot, Kramer, Simons, Fabiani, \& Gratton, 2008); and that novice athletes were not elites in any other sport. All athletes were native French speakers.

All participants were tested in accordance with local laws and regulations, as well as with American Psychological Association standards of ethics. To ensure confidentiality, subjects were informed that all data set that could identify them would be replaced by participants' codes.

Materials and procedure

We used the French version of the original MIQ-R (Lorant \& Nicolas, 2004), the MIST and the MRT to assess imagery ability. These three tasks, as well as their specific testing and scoring procedures, are detailed below:

Movement Imagery Questionnaire-Revised (MIQ-R, Hall \& Martin, 1997)

We used the French version of the MIQ-R, relevant translation of the eight items questionnaire and its directions, which has been found to be both valid and reliable (Lorant \& Nicolas, 2004). There was no time constraint for this test. Participants were aware that they could take as long as they needed in order to complete the questionnaire. We asked them to execute a movement, and then imagine it either visually ('attempt to see yourself making the movement just performed...') or kinesthetically ('attempt to feel yourself making the movement just performed...'). They were asked to rate the quality of the imagined movements on a seven-point scale varying from 'very hard to see/to feel' (one) to 'very easy to see/to feel' (seven). We obtained a total score by summing all their responses. Therefore, the 
maximum score was 56. However, and for further analysis, we also differentiated visual and kinesthetic scores, by summing the respective visual or kinesthetic items. Consequently, visual and kinesthetic could range from 4 to 28 .

Movement Imagery Specific Test (MIST, Moreau, Mansy-Dannay, Clerc, \& Guerrien, under review)

Three different versions of the MIST exist, presenting different items depending on the sport speciality (fencing, judo or wrestling). Each test is based on twenty verbal items, each describing an original situation, and involving production, transformation and retention of spatial patterns specific to each sport. From that situation, a particular answer is expected (see table 1). There never is more than one correct answer per item. We ensured that the situations described were highly meaningful for all athletes, including novices. Thus, we wanted to avoid yielding any differences between elites and novices based on their understanding of the task. Although cognitive efforts required to solve each problem can be challenging, these items are fairly easy to picture for the purpose of selecting the best and quickest answers. We considered this factor prior to the experiment by working on different versions of the test with competent national coaches from the three sports concerned. Therefore, the stimuli are not visual but verbal, because we wanted to avoid any mistakes arising by using visual indefinite pictures to represent athletes in action. Having sentences with an international and well-known terminology commonly used by athletes during their acquisition and learning processes avoids any unsettling confusion. No particular instruction was provided concerning the perspective (first or third person) and the modality (visual or kinesthetic) to be used by the participants. Furthermore, we tried to optimize the time constraint. For that purpose, we first tested some participants who were not involved in the experiment (pilot study), in order to specify the length of the test. Regarding this pilot study, we decided to give three minutes to solve this twenty-item task. We scored the test giving one point for each correct answer and zero point when the answer was blank or wrong. Consequently, the maximum possible score was 20. The MIST has been standardized, and its validity and reliability have been confirmed in a previous study (Moreau, Mansy-Dannay, Clerc, \& Guerrien, under review). 
Table 1: Examples of items for each version of the MIST.

\section{MIST Fencing}

Starting from an engagement in sixte with my opponent whose left hand is armed. If I am performing a lunge, am I in his/her parry of quarte or sixte?

口 Quarte

$\square$ Sixte

\section{MIST Judo}

I am performing juji-gatame on my opponent's right arm. Which of my legs is holding his head down?

$\square$ Right

$\square$ Left

\section{MIST Wrestling}

I am performing an ankle lace to my opponent's left. Which of his ankles is on the top of the other?

$\square$ Right

$\square$ Left

Mental Rotation Test (MRT, Vandenberg \& Kuse, 1978)

We used a printed version of the MRT, including two sets of ten problems each, based on Shepard and Metzler (1971) stimuli (two different versions of this test exist in the literature, one with 20 and the other with 24 items). We limited the testing time to three minutes for each set of 10 problems, separated by a five-minute break. According to Albaret and Aubert (1996), we gave two points for two right answers, one point if the athlete answered just half of the item and the answer was correct, and zero point if there was one or two mistake(s). We chose not to collect reaction times, since this variable was not relevant for comparison between different tasks and designs.

Participants performed each task in a quiet room and were given detailed and standardized instructions about the three tests prior to each session. They took the Mental Rotation Test first, in order to avoid inducing embodied solving strategies by contamination from any of the two other tests. Indeed, research has shown that when instructions emphasize on endogenous motor strategies for solving mental rotation involving geometric shapes, premotor and motor cortex activation was found, but not when participants were asked to use exogenous strategies (Kosslyn, 
Thompson, Wraga, \& Alpert, 2001). Thus, using body-related items might result in motor cortex activation in a subsequent mental rotation object-based task (Wraga, Thompson, Alpert, \& Kosslyn, 2003).

\section{Results}

We used different statistical tools in order to yield relevant features of the MIQ-R, the MIST and the MRT. We present descriptive statistics, analyses of variance, and finally correlation and regression analyses in this section.

Descriptive Statistics

Descriptive statistics are displayed in table 2. Elite athletes performed better than novices regardless of the task. This is especially visible by comparing performances in the MRT and the MIST. High standard deviations in MRT scores for elites and for the whole sample are due to gender differences.

Table 2: Summary of descriptive statistics for elite and novice groups and for the whole sample

\begin{tabular}{lcccccc}
\hline \multicolumn{1}{c}{ Level } \\
\multicolumn{1}{c}{ Elite } & \multicolumn{2}{c}{ Novice } & \multicolumn{2}{c}{ All } \\
\hline \multicolumn{1}{c}{ Task } & Mean & SD & Mean & SD & Mean & SD \\
\hline & & & & & & \\
MRT & 23.7 & 8.97 & 11.7 & 3.44 & 17.5 & 8.94 \\
MIST & 16.2 & 2.99 & 7.0 & 1.83 & 11.6 & 5.25 \\
MIQ-R & 50.3 & 3.68 & 42.4 & 6.67 & 46.4 & 6.65 \\
\hline
\end{tabular}

A better perspective on MRT, MIST and MIQ-R performances, for each group (elite males, elite females, novice males, novice females), can be inferred from figure 1. 


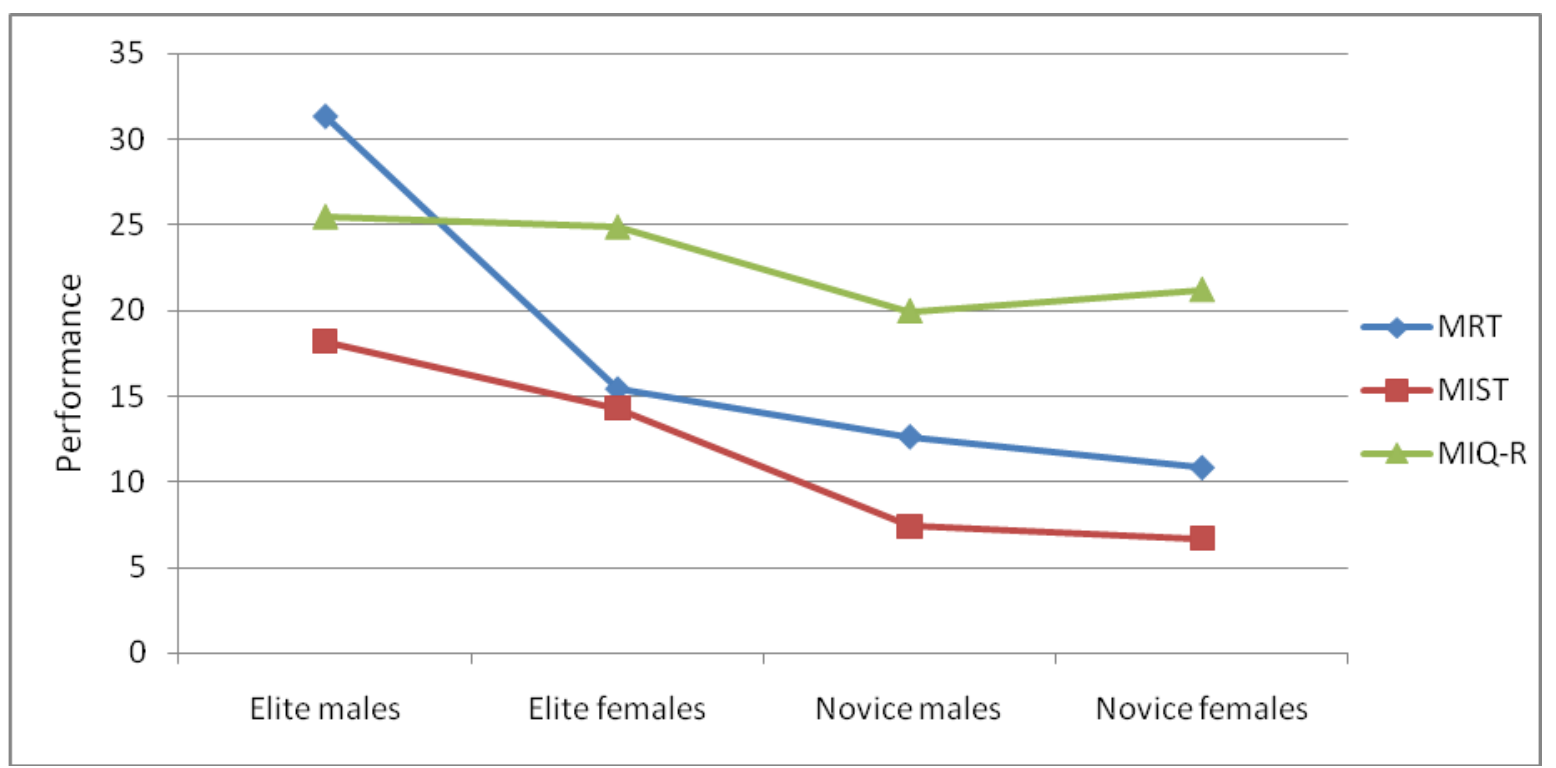

Figure 1: MRT, MIST and MIQ-R performances depending on expertise and gender variables.

Analyses of variance

We specified previous findings by conducting an ANOVA for each test. Prior to these statistical analyses, assumptions on normality and on the homogeneity of variances were verified for each sample (Kolmogorov-Smirnov and Levene's tests being nonsignificant, in all cases).

$M I Q-R$. The interaction between expertise and gender variables did not reach significance value $(F(1,48)=.24, p=.62)$. These results contrast with MIST and MRT data presented below.

MIST. Elite athletes scored better than novices regardless of gender $(F(1,48)=309,03$, $\left.p<.001, \eta^{2}=0,87\right)$. The difference males-females was significant $(F(1,48)=19,88, p<.001$, $\left.\eta^{2}=0,29\right)$, although it is important to consider the difference between elites and novices as well, since the level by gender interaction was significant $(F(1,48)=9,35$, $\left.p<.05, \eta^{2}=0,16\right)$. Thus, elite males $(M=18.2 ; S D=1.70)$ performed constantly higher than elite females $(M=14.3 ; S D=2.71)$, regardless of sport (Tukey's test significant, $p<.001)$.

MRT. The MRT showed similar results. The ANOVA yielded a main effect of expertise $\left(F(1,48)=134.54, p<.001, \eta^{2}=0.74\right)$, and a main effect of gender $(F(1,48)=77.71, p<.001$, $\left.\eta^{2}=0.62\right)$. Besides, the interaction between expertise and gender revealed a stronger effect of expertise for males than females $\left(F(1,48)=49.36, p<.001, \eta^{2}=0.51\right)$. 
Correlation matrices and regression analyses

We conducted correlation and regression analyses in order to specify the relation between the three tests considered.

Correlation matrices on the elite group showed non-significant values of $r$ for $M I Q-R$ and MRT variables $(r(30)=.13$, n.s.) and for MIQ-R and MIST variables $(r(30)=.050$, n.s.). When we separated the visual and the kinesthetic components, we still obtained non-significant $r$ values $(r(30)=.12$, n.s., for MIQ-R Visual/MRT; $r(30)=-.005$, n.s., for MIQ-R Visual/MIST; $r(30)=.09$, n.s., for MIQ-R Kinesthetic/MRT; and $r(30)=.11$, n.s., for MIQ-R Kinesthetic/MIST). However, the correlation between MRT and MIST was significant $(r(30)=.79, p<.001)$.

Analyses performed on the novice group yielded similar results. Correlation matrices on this group showed non-significant values of $r$ for MIQ-R and MRT variables $(r(30)=-$ .11 , n.s.) and for MIQ-R and MIST variables $(r(30)=-.35$, n.s.). Even when the visual and the kinesthetic components were processed separately, we noticed nonsignificant $r$ values $(r(30)=-.09$, n.s., for MIQ-R Visual/MRT; $r(30)=-.35$, n.s., for MIQ-R Visual/MIST; $r(30)=-.12$, n.s., for MIQ-R Kinesthetic/MRT; and $r(30)=-.29$, n.s., for MIQ-R Kinesthetic/MIST). However, and as for the elite group, the correlation between MRT and MIST was significant $(r(30)=.48, p<.001)$.

Results of the correlation analyses led us to perform multiple regression analyses on the data. A summary of regression analyses for variables predicting MIQ-R performance is displayed in table 3. Neither the MIST nor the MRT was a significant predictor of the MIQ-R performance, regardless of the subgroups considered. These results will be discussed in the next section.

Table 3: Summary of regression analyses for variables predicting MIQ-R performance

\begin{tabular}{cccccc}
\hline Variable & $\beta$ & SE $\beta$ & $R^{2}$ & $F$ & $P$ \\
\hline Elites & & & & & \\
MIST & .050 & .189 & .002 & .07 & .79 \\
MRT & .129 & .187 & .017 & .47 & .49 \\
Novices & & & & & \\
MIST & -.346 & .177 & .120 & 3.81 & .06 \\
MRT & -.112 & .187 & .013 & .36 & .55 \\
\hline
\end{tabular}




\section{Discussion and conclusion}

The main aim of this research was to compare the relevance of self-report questionnaires, widely use in sport's research, and performance-based tests to assess movement imagery ability in sports. Particular results gathered for that purpose provide us with several key-points to answer the issue.

First of all, we did not find any correlation between the MIQ-R and the MRT. In fact, this could be expected since a large body of research has shown that imagery assessed via self-report questionnaires and spatial abilities assessed through performance-based tests are unrelated, as detailed previously in this paper. Indeed, our findings add to the growing amount of literature differentiating these abilities in the field of visuospatial cognition.

Second, we did not observe any correlation between the MIQ-R and the MIST. Although this is consistent with the distinction between questionnaires and performance-based tests, it should be noted that the MIST items are sport-specific, as opposed to the previously discussed MRT items (geometric shapes). Thus, the presentation modalities in the MIQ-R and in the MIST are similar, that is, both tests imply recoding movements or successions of movements from verbal sentences describing a specific situation. One of the differences lies within the response modality, self-report or actual accurate answer, respectively. Another distinction is the specificity of the tests. Although providing a MIST version for every sport or activity might be difficult, assessing movement in elite athletes through the MIQ-R containing general and sport-unrelated items can only generate rough insights into athletes' motor representations, hence not particularly meaningful for coaches and instructors.

However, we found a significant correlation between the MIST and the MRT (both performance-based tests), the former assessing movement imagery and the latter assessing mental rotation. This means that what is being measured by both tests shares a common variance, implying that they tap into similar processes, at least partly, or that they are influenced by a common factor. Thus, both of these tests showed a significant positive effect of expertise level in sport, elite athletes scoring higher, tending to prove that abilities measured through these tests are more important and preeminent for actual motor execution and performance.

Furthermore, MIST results show substantial differences between elite and novice athletes. These differences were expected since the task is highly related to their everyday practice. We also found significant gender differences for elites but not for 
novices. These results were found with MRT performance as well, supporting research on spatial ability and mental rotation which favors males in almost any experimental design (see Voyer, Voyer, \& Bryden, 1995; and Peters et al., 1995, for reviews). However, these differences were not visible in MIQ-R results. Elite athletes seem to be more severe and strict regarding their introspective experience. Indeed, they did not outscore novices. This might occur because they actually know what a high level of imagery would 'look like' or 'feel like', and so they might consider, for example, "somewhat easy to see/feel" what novices might refer as "very easy to see/feel". Besides, some novices might have no precise idea of what is a clear and relevant mental image, even though they might try to be as objective as possible. Consequently, they may judge themselves competent in their own subjective referential, although their mental images may be poor and inefficient.

Altogether, these results provide further evidence for dissociation between imagery ability traditionally assessed through self-report questionnaires, and spatial ability experimental measurements involving performance-based tests. In fact, assessing imagery ability exclusively through self-reports seem irrelevant to us, for the subjective variable induced via introspection cannot be controlled. However, we do not argue here for rejecting this range of testing tools, but we believe that what is being measured by them is sometimes mistaken. One possible answer to that matter would be to pair them with self-esteem questionnaires, in order to control the introspective parameter in the experimental design.

Moreover, performance-based tests such as those presented in this paper bring up a few problems as well. Thus, they imply a non-direct measure of spatial abilities and imagery ability, through responses being correct or not. Cognitive processes involved to perform well in these tasks, as well as strategies used, might vary from one participant to another (Just \& Carpenter, 1985), which has already raised some issues in the visuospatial cognition field. This can be partly controlled by asking participants about which particular strategies they used to perform a task, and compare them with their performance, along with completing such data by neuroimaging techniques.

In fact, more research involving imagery and spatial ability is needed to precise the relationship between the former and the latter, and to reveal and define their distinctions and similarities, in order to build a comprehensive model of human spatial abilities and imagery processes. For example, correlations data between various spatial tasks and imagery tests, as well as results from the neurosciences research, should allow a better insight into that particular field. 
In conclusion, spatial abilities can include imagery assessed through self-report questionnaires as a distinguished category, but emphasis has to be put on comparing similar items modalities (Burton \& Fogarty, 2003), and also on providing complementary measurements, such as self-confidence questionnaires, in order to control part of the subjective trait inherent to this kind of tests. Besides, within the movement imagery paradigm these questionnaires should be supplemented by different modalities of measures, such as performance-based tests, to ensure an objective and complete assessment of one's ability to generate, maintain and manipulate motor images.

\section{References}

Atienza, F., Balaguer, I., \& Garcia-Merita, M. L. (1994). Factor analysis and reliability of the movement imagery questionnaire. Perceptual and Motor Skills, 78, 1323-1328.

Albaret, J.M., \& Aubert, E. (1996). Etalonnage 15-19 ans du test de rotation mentale de Vandenberg, Evolutions psychomotrices, 34(8), 276-279.

Boot, W. R., Kramer, A. F., Simons, D. J., Fabiani, M., \& Gratton, G. (2008). The effects of video game playing on attention, memory, and executive control. Acta Psychologica, 129, 387-398.

Burton, L. J., \& Fogarty, G. J. (2003). The factor structure of visual imagery and spatial abilities. Intelligence, 31, 289-318.

Dean, G. M., \& Morris, P. E. (1991). Imagery and spatial ability: when introspective reports predict performance. In R. H. Logie, \& M. Denis (Eds.), Mental images in human cognition ( pp. 331-347). North-Holland: Elsevier.

Decety, J. (2002). Is there such a thing as functional equivalence between imagined, observed, and executed action? In A. N. Meltzoff \& W. Prinz (Eds.), The imitative mind: Development, evolution, and brain bases (pp. 291-310). Cambridge, England: Cambridge University Press.

Driskell, J. E., Copper, C. \& Moran A. (1994). Does mental practice enhance performance? Journal of Applied Psychology, 79, 4, 481-492.

Feltz, D. L. \& Landers, D. M. (1983). The effects of mental practice on motor skill learning and performance: A meta-analysis, Journal of Psychology, 5, 25-57. 
Goss, S., Hall, C., Buckolz, E., \& Fishburne, G. (1986). Imagery ability and theacquisition and retention of movements. Memory and Cognition, 14(6), 469-477.

Grezes, J. \& Decety, J. (2001). Functional anatomy of execution, mental simulation, observation and verb generation of actions: A meta-analysis. Human Brain Mapping, 12: $1-19$.

Hall, C, Buckolz, E, \& Fishburne, G. (1989). Searching for a relationship between imagery ability and memory of movements. Journal of Human Movement Studies, 17, 89-100.

Hall, C. \& Martin, K. (1997). Measuring movement imagery abilities: a revision of the Movement Imagery Questionnaire. Journal of Mental Imagery, 21 (1 \&2), 143-154.

Hall, C. \& Pongrac, J. (1983). Movement Imagery Questionnaire. London, Ontario : University of Western Ontario.

Hall, C., Pongrac, J., \& Buckolz, E. (1985). The measurement of imagery ability. Human Movement Science, 4, 107-118.

Halpern, D. F. \& Collaer, M. L. (2005). Sex differences in Visuospatial Abilities: More than Meets the Eye. In Shah, P. \& Miyake, A. The Cambridge Handbook of Visuospatial Thinking (pp. 170-212). Cambridge University Press, New York.

Hegarty, M. \& Waller, D.A. (2005). Individual differences in spatial abilities. In Shah, P. \& Miyake, A. The Cambridge Handbook of Visuospatial Thinking (pp. 121-169). Cambridge University Press, New York.

Isaac, A. R., Marks, D. F., \& Russell, D. G. (1986). An instrument for assessing imagery of movement: The vividness of movement imagery questionnaire (VMIQ). Journal of Mental Imagery, 10, 23-30.

Jansen, P., Titze, C., \& Heil, M. (2009). The influence of juggling on mental rotation performance. International Journal of Sport Psychology, 40(2), 351-359.

Jeannerod, M., (1994). The representing brain: neural correlates of motor intention and imagery. Behavioral Brain Sciences, 17, 187-245.

Jeannerod, M. (1995). Mental imagery in the motor context, Neuropsychologia, 33, 14191433. 
Jeannerod, M., (2001). Neural simulation of action: a unifying mechanism for motor cognition. Neurolmage 14, 103-109.

Just, M. A., \& Carpenter, P.A. (1985). Cognitive coordinate systems: Accounts of mental rotation and individual differences in spatial ability. Psychological Review, 92, 137-172.

Kirasic, K.C. \& Allen, G.L. (1985). Aging, Spatial Performance and Spatial Competence. In: Charness, N. (Ed.). Aging and Human Performance. JohnWiley \& Sons, Ltd, pp. 191223.

Kosslyn, S. M., Brunn, J., Cave, K. R., \& Wallach, R. W. (1984). Individual differences in mental imagery ability: a computational analysis. Cognition, 18, 195-243.

Kosslyn, S. M., Thompson, W. L., Wraga, M., \& Alpert, N. M. (2001). Imagining rotation by endogenous versus exogenous forces: Distinct neural mechanisms. NeuroReport, 12, 2519-2525.

Leone, G., Taine, M., \& Droulez, J. (1993). The influence of long-term practice on mental rotation of 3-D objects. Cognitive Brain Research, 1 (4), 241-255.

Lohman, D., \& Nichols, P. (1990). Training spatial abilities: Effects of practice on rotation and synthesis tasks. Learning and Individual Differences, 2(1), 67-93.

Lorant, J. \& Nicolas, A. (2004). Validation de la traduction française du Movement Imagery Questionnaire-Revised (MIQ-R). Science \& Motricité, 53, 57-68.

Lorey, B., Pilgramm, S., Walter, B., Stark, R., Munzert, J., \& Zentgraf, K. (2010). Your mind's hand: Motor imagery of pointing movements with different accuracy. Neurolmage, 49(4), 3239-3247.

Moreau, D., Mansy-Dannay, A., Clerc, J., \& Guerrien, A. (2010). Présentation d'un outil original mesurant la qualité des représentations motrices en sport de combat : le Test Spécifique d'Imagerie du Mouvement (TSIM). Under review.

Moreau, D., Mansy-Dannay, A., Clerc, J., \& Guerrien, A. (submitted). Spatial ability and motor performance: Assessing mental rotation processes in elite and novice athletes.

Morris, T., Spittle, M., \& Watt, A. P. (2005). Imagery in sport. Champaign, IL: Human Kinetics. 
Peters, M., Laeng, B., Latham, K., Jackson, M., Zaiyouna, R., \& Richardson, C. (1995). A redrawn Vandenberg and Kuse Mental Rotations Test: Different versions and factors that affect performance. Brain and Cognition, 28, 39-58.

Poltrock, S. E., \& Agnoli, F. (1986). Are spatial visualisation ability and visual imagery ability equivalent? In R. J. Sternberg (Ed.), Advances in the psychology of human intelligence, vol. 3 ( pp. 255-296). Hillsdale, NJ: Lawrence Erlbaum.

Richardson, A. (1977). The meaning and measurement of memory imagery. British Journal of Psychology, 68, 29-43.

Richardson, A. (1983). Imagery: definition and types. In A. A. Sheikh (Ed.), Imagery: current theory, research, and application ( pp. 3-42). New York: Wiley.

Roure, R., Collet, C., Deschaumes-Molinaro, C., Dittmar, A., Rada, H., Delhomme, G., \& Vernet-Maury, E. (1998). Autonomic nervous system responses correlate with mental rehearsal in volley-ball training, European Journal of Applied Physiology, 78, 99-108.

Schroeder, D.H. \& Salthouse, T.A. (2004). Age-related effects on cognition between 20 and 50 years of age. Personality and Individual Differences. 36, 393-404.

Shepard, R.N., \& Metzler J. (1971). Mental rotation of three dimensional objects. Science, $171,701-703$.

Vandenberg, S.G., \& Kuse A.R. (1978). Mental rotations, a group test of threedimensional spatial visualization. Perceptual and Motor Skills, 47, 599-604.

Voyer, D., Voyer, S., \& Bryden, M.P. (1995). Magnitude of sex differences in spatial abilities: A meta-analysis and consideration of critical variables. Psychological Bulletin, $117,250-270$.

Weinberg, R. (2008). Does imagery work? Effects on performance and mental skills. Journal of Imagery Research in Sport and Physical Activity, 3(1).

Wraga, M., Thompson, W. L., Alpert, N. M., \& Kosslyn, S. M. (2003). Implicit transfer of motor strategies in mental rotation. Brain and Cognition, 52, 135-143.

Yaguez, L., Nagel, D., Hoffman, H., Canavan, A. G. M., Wist, E., \& Homberg, V. (1998). A mental route to motor learning: Improving trajectorial kinematics through imagery. Behavioral Brain Research, 90(1), 95-106. 
About the Authors:

David Moreau is currently a Ph.D. Candidate in Cognitive Psychology at the University of Lille 3, working on spatial abilities, mental imagery, and working memory. $\mathrm{He}$ is also a Fulbright Program Alumnus, and has been teaching and doing research projects in different universities, in Europe and in the US.

Address for correspondence: David Moreau, EA 4072 PSITEC, University of Lille 3, Lille, France

E-mail: david.moreau@fulbrightmail.org

Jérôme Clerc is a Senior Lecturer in Cognitive Psychology at the University of Lille 3.

Annie Mansy-Dannay is a Senior Lecturer in Cognitive Psychology at the University of Lille 3 and the University of Lille 2.

Alain Guerrien is a Professor of Cognitive Psychology at the University of Lille 3. 